\title{
EL SISTEMA DE EVALUACIÓN DE IMPACTO AMBIENTAL ANÁLISIS DEL DISEÑO DE FISCALIZACIÓN Y SU CUMPLIMIENTO EN LA REGIÓN DEL BÍO-BÍO*
}

\author{
Jaime Lamas y Carlos Chávez
}

\begin{abstract}
Este trabajo revisa el diseño del Programa de Fiscalización Ambiental Regional y evalúa los resultados de cumplimiento de las Resoluciones de Calificación Ambiental (RCA) para proyectos sometidos al Sistema de Evaluación de Impacto Ambiental (SEIA). Nuestro análisis utiliza una base de datos que contiene información de fichas de fiscalización de 204 visitas de inspección desarrolladas bajo la coordinación de la Conama de la región del Bío-Bío, entre 1999 y el primer semestre del año 2004. Los resultados indican que el nivel de cumplimiento perfecto de la RCA alcanza sólo al 50\% de los proyectos inspeccionados, en tanto que la incidencia de cumplimiento
\end{abstract}

Jaime Lamas. Magíster (c) en Economía de Recursos Naturales y del Medio Ambiente, Universidad de Concepción. Profesional de la Comisión Nacional del Medio Ambiente, región del Bío-Bío. Email: jlamas.8@conama.cl

Carlos Chávez. Doctor of Philosophy (Ph. D.) Economía Ambiental y Recursos Naturales, University of Massachusetts, Amherst, Estados Unidos. Profesor asociado, Departamento de Economía, Universidad de Concepción. Email: cchavez@udec.cl

* Agradecemos a la Comisión Nacional del Medio Ambiente de la Región del Bío-Bío, particularmente al Programa de Fiscalización Ambiental Regional, por facilitar el acceso a la información de fiscalización. 
bajo o incumplimiento total afecta a un $20 \%$ de los proyectos fiscalizados. Se presenta un perfil de los proyectos detectados en incumplimiento y se discuten recomendaciones tendientes a perfeccionar el diseño de fiscalización que permita mejorar los niveles de cumplimiento ambiental en el SEIA.

\section{INTRODUCCIÓN}

E el transcurso de las últimas décadas, Chile ha experimentado un proceso de crecimiento económico sostenido, el cual ha estado fuertemente ligado al desarrollo de actividades productivas de sectores exportadores. Una característica común en estas actividades es su orientación al uso intensivo de recursos naturales renovables y no renovables y del medio ambiente en general. El proceso mencionado ha ejercido una considerable presión sobre algunos recursos naturales y ambientales. Dicha presión se ha expresado a través de los impactos ambientales que se han ido generando por la ejecución de diversos tipos de proyectos que originan contaminación y otras consecuencias negativas sobre los ecosistemas en los que se insertan (Evaluaciones del Desempeño Ambiental, Chile, OCDE-CEPAL, 2005).

Como respuesta a las demandas de un manejo adecuado de los recursos naturales y el medio ambiente, y particularmente desde inicios de los años noventa, el Estado chileno ha buscado dar solución a sus problemas ambientales mediante un modelo de coordinación ambiental multisectorial. Un hito importante en dicho proceso lo constituye la promulgación de la Ley 19300, denominada Ley de Bases Generales del Medio Ambiente, la que entró en vigencia en marzo del año 1994. La referida ley considera, entre otros aspectos relevantes, la creación de un Sistema de Evaluación de Impacto Ambiental (SEIA), mediante el cual se evalúan los aspectos ambientales de los proyectos de inversión que se ejecutan en el país. Dicho sistema de evaluación entró en vigencia el año 1997 con la publicación oficial del Reglamento del Sistema de Evaluación de Impacto Ambiental (RSEIA) ${ }^{1}$.

El instrumento de regulación ambiental que ha alcanzado la mayor relevancia en Chile es el SEIA. Mediante éste se estableció la obligación de someter a evaluación de impacto ambiental a los proyectos de inversión que se desarrollan en el país y que puedan causar impacto ambiental en cual-

${ }^{1}$ El RSEIA fue publicado mediante Decreto Supremo No 30, en marzo de 1997, y posteriormente modificado por el Decreto Supremo No 95 de agosto del año 2001. 
quiera de sus fases. Consecuentemente, el SEIA se constituye en el principal instrumento preventivo de gestión ambiental con que cuenta el país. Por una parte, a través de él las empresas se comprometen a cumplir con las regulaciones ambientales vigentes bajo las cuales se aprueban los proyectos. Por otra parte, el Estado, a través de sus diferentes órganos, es el encargado de hacer cumplir dichas regulaciones ambientales. Dos son los instrumentos de que dispone el Estado para tal propósito: primero, el desarrollo de visitas de inspección con el propósito de detectar el estado de cumplimiento de los proyectos evaluados con las regulaciones y compromisos contraídos por sus representantes, y, segundo, la imposición de multas o sanciones en aquellos casos en que se detectan incumplimientos $^{2}$.

La literatura que trata de la implementación de las regulaciones ambientales y del comportamiento de los agentes sujetos a ellas sugiere que la decisión de cumplimiento depende de diferentes factores, entre los que se incluyen aquellos referidos a la capacidad del regulador (o fiscalizador, si es independiente) para elaborar y ejecutar programas de fiscalización eficientes, como también los costos de las firmas u otros agentes regulados asociados al cumplimiento de las exigencias ambientales (ver por ejemplo Cohen, 1999; Dasgupta et al., 2000, y Heyes, 2000). Considerando la diversidad de agentes sujetos a regulaciones ambientales, así como las diferencias que éstos presentan, es posible esperar que el grado de cumplimiento individual sea variable y, en muchos casos, posiblemente bajo, en especial en los países en vías de desarrollo (World Bank, 2002). De hecho, entre los problemas que enfrentan las autoridades responsables de la implementación de políticas de regulación ambiental en países en desarrollo están la disponibilidad limitada de personal especializado y también los presupuestos normalmente restringidos para el desarrollo de actividades de fiscalización para inducir cumplimiento (Palacios y Chávez, 2005). En el caso de nuestro país, se ha reconocido recientemente que el diseño de fiscalización para inducir cumplimento de las normativas es uno de los puntos sensibles de la política ambiental nacional. Específicamente, entre una serie de recomendaciones sugeridas con el propósito de mejorar la gestión ambiental de nuestro país se ha indicado la necesidad de “...examinar formas de fortalecer la capacidad de cumplimiento y fiscalización” para el desarrollo y fortalecimiento de los

${ }^{2}$ El RSEIA establece que corresponderá a los organismos del Estado que, en uso de sus facultades legales, participan en el SEIA fiscalizar el permanente cumplimiento de las normas y condiciones sobre la base de las cuales se aprobó el proyecto. 
marcos normativos destinados a la protección ambiental y el manejo adecuado de los recursos naturales (OCDE-CEPAL, 2005, pp. 18 y 124) ${ }^{3}$.

El desarrollo de estrategias y acciones de fiscalización ambiental para inducir cumplimiento de normativas ambientales es una tarea compleja que demanda recursos. Las limitaciones para dicha tarea obedecen, por un lado, a razones económicas y, por otro lado, a la presencia de problemas de asimetrías de información entre agencia o unidad fiscalizadora y agente regulado ${ }^{4}$. Es posible esperar que agentes regulados tiendan a evitar cumplir con las regulaciones ambientales debido a que la vigilancia destinada a detectar infracciones es esporádica, y a que las sanciones y otras acciones disuasivas resultan en muchos casos no sólo mínimas sino que no se corresponden con la magnitud de la infracción (Dasgupta et al., 2000).

No obstante la relevancia de la fiscalización para asegurar niveles adecuados de cumplimiento, en el caso de Chile y de la región del Bío-Bío en particular, lamentablemente no se ha realizado una revisión sistemática de los resultados de cumplimiento de las exigencias ambientales por parte de los agentes que han obtenido la aprobación ambiental de sus proyectos evaluados bajo el SEIA ${ }^{5}$. De esta forma, nuestro trabajo constituye, de

${ }^{3}$ Es interesante mencionar que, no obstante que compartimos esta sugerencia, en nuestra opinión el informe de desempeño ambiental de Chile antes referido no ofrece evidencia respecto de incumplimientos de programas de regulación ambiental específicos. Debido a ello entendemos que, muy probablemente, la sugerencia planteada en relación con mejorar la fiscalización ambiental está basada en la revisión general del diseño institucional más que en la evaluación de estrategias de fiscalización específicas y/o de los resultados de cumplimiento relacionado con regulaciones o normativas referidas a la protección ambiental y adecuado manejo de recursos naturales. En este sentido, nuestro trabajo contribuye con evidencia específica que tiende a apoyar la referida recomendación.

${ }^{4}$ La fiscalización de regulaciones ambientales incluye el conjunto de acciones desarrolladas por agencias de gobierno y otros, las que son destinadas a inducir cumplimiento de los agentes bajo regulación y a corregir situaciones que puedan poner en peligro el medio ambiente o la salud de las personas. Dichas acciones comprenden inspecciones o auditorías con el propósito de detectar violaciones, negociaciones con agentes individuales o representantes de empresas detectados en incumplimiento con el propósito de generar acuerdos conducentes al cumplimiento, y la aplicación de sanciones o el desarrollo de acciones legales en el caso de que una violación sea descubierta (ver U. S. EPA, 1992). En este sentido, utilizamos el término "fiscalización" como sinónimo de la palabra inglesa enforcement.

${ }^{5}$ La literatura existente a nivel nacional relacionada más directamente al análisis de la fiscalización y cumplimiento de regulaciones ambientales es escasa. En efecto, un análisis de aspectos referidos al diseño de fiscalización y los factores determinantes de cumplimiento individual de fuentes que participan en el Programa de Compensación de Emisiones de la ciudad de Santiago se encuentra en Palacios y Chávez (2002) y (2005). Más recientemente, Ruiz-Tagle (2006) estudia los factores determinantes del cumplimiento de regulaciones ambientales en la industria manufacturera nacional. Este 
acuerdo a nuestro conocimiento, un esfuerzo inicial respecto al análisis del funcionamiento del Programa de Fiscalización Ambiental Regional (PFAR) y al grado de cumplimiento por parte del sector privado ${ }^{6}$.

El objetivo de este trabajo es proveer una revisión del diseño del Programa de Fiscalización Ambiental Regional (PFAR) y una evaluación de los resultados en términos de cumplimiento ambiental de proyectos aprobados bajo el Sistema de Evaluación de Impacto Ambiental (SEIA) en la región del Bío-Bío, en el período 1999-2004. El trabajo analiza, desde el punto de vista técnico, alternativas para perfeccionar el Programa de Fiscalización y a la vez aporta antecedentes a fin de perfeccionar los incentivos que permitan mejorar los niveles de cumplimiento bajo el SEIA.

Nuestro análisis se sustenta en una base de datos construida a partir de información existente en las fichas de fiscalización generadas por las visitas de inspección desarrolladas bajo el PFAR en la región del BíoBío. Con el propósito de lograr los objetivos planteados, se construyó una base de datos que incluye información detallada recopilada a partir de la revisión individual de las fichas de inspección correspondientes a 204 visitas de fiscalización realizadas por el Departamento de Fiscalización Ambiental de la Conama de la región del Bío-Bío, en el período comprendido entre 1999 y el primer semestre de 2004. Esta información fue complementada con la revisión de otras fuentes secundarias de información que ayudaron a caracterizar tanto el nivel de exigencias ambientales que enfrentaban los proyectos considerados como también otras características de los proyectos fiscalizados.

El trabajo se organiza según se describe a continuación. En la sección 2 se presenta una descripción detallada del diseño regulatorio considerado en el Sistema de Evaluación de Impacto Ambiental (SEIA). En la sección 3 se describe el funcionamiento del SEIA y de su fiscalización para el cumplimiento de la Resolución de Calificación Ambiental (RCA) en la región del Bío-Bío. La sección 4 presenta los resultados del análisis de cumplimiento individual de los proyectos que, contando con su RCA, fue-

trabajo examina, entre otros, el rol de mecanismos formales e informales de regulación en la decisión de cumplimiento individual. Entre los mecanismos informales de regulación, el trabajo considera el efecto de la acción de comunidades locales o grupos de ciudadanos organizados, y de agentes individuales en su rol de inversionistas y consumidores, sobre la decisión de cumplimiento de establecimientos manufactureros. Lamentablemente, ninguno de estos trabajos considera el diseño de fiscalización y cumplimiento en el marco del SEIA.

${ }^{6}$ Se entiende por PFAR el proceso administrativo y práctico coordinado intersectorialmente cuyo objetivo es constatar el cumplimiento ambiental, de acuerdo con las exigencias establecidas en la RCA. 
ron fiscalizados durante el período 1999-2004 en la región del Bío-Bío. Finalmente, en la sección 5 se presenta un análisis crítico de los aspectos referidos al diseño de fiscalización para inducir cumplimiento de la RCA en el marco del SEIA, y se discuten sugerencias para mejorar el cumplimiento ambiental en la región del Bío-Bío.

\section{SISTEMA DE EVALUACIÓN DE IMPACTO AMBIENTAL}

Esta sección describe y analiza aspectos de diseño general del Sistema de Evaluación de Impacto Ambiental y su funcionamiento. La sección está divida en dos partes. Primero: se revisa brevemente el marco general de la regulación ambiental basado en el SEIA vigente en Chile. Segundo: se describen los procedimientos para el proceso de evaluación de impacto ambiental considerados por el SEIA y su Reglamento.

\subsection{Marco general para la regulación ambiental en Chile basada en el SEIA}

El SEIA tiene como objetivos detectar potenciales impactos ambientales negativos de los proyectos o actividades nuevos que se pretenden ejecutar y, al mismo tiempo, adoptar las medidas necesarias para evitarlos, minimizarlos y, por último, buscar las reparaciones ambientales adecuadas. El diseño general del SEIA considera procedimientos no sólo para establecer condiciones de ejecución de proyectos, sino que también contempla que las empresas (titulares de los proyectos) se comprometan a cumplir con las regulaciones ambientales vigentes bajo las cuales se aprobó el proyecto de inversión sometido a evaluación.

Durante el año 1997, mediante el Decreto Supremo No 30, entró en vigencia el Reglamento del Sistema de Evaluación de Impacto Ambiental (RSEIA). (El referido reglamento sería posteriormente modificado por el Decreto Supremo $N^{\circ} 95$ de agosto del año 2001.) El Reglamento del SEIA estableció la obligación de ingresar a evaluación los proyectos de inversión que se desarrollan en el país y que puedan causar impacto ambiental en cualquiera de sus fases ${ }^{7}$.

${ }^{7}$ El Reglamento señala de manera detallada los tipos de proyectos que deben ser evaluados ambientalmente, indicando dimensiones mínimas de los mismos, según sea el caso. Establece además los contenidos básicos de un Estudio de Impacto Ambiental (EIA) o de una Declaración de Impacto Ambiental (DIA) y los procedimientos a seguir para obtener un pronunciamiento favorable de la autoridad. Adicionalmente, el Reglamento establece y regula las medidas de mitigación, reparación y/o compensación que eventualmente deben implementarse para hacer ambientalmente más amigable un pro- 
En el funcionamiento del SEIA la Dirección Regional de Conama desempeña el rol de coordinar la evaluación ambiental de los proyectos sometidos al SEIA ${ }^{8}$. En efecto, La Dirección Regional es la encargada de elaborar informes (solicitud de aclaraciones al titular, reuniones del comité revisor del proyecto, informe consolidado de evaluación ambiental), es decir, realiza el trabajo técnico que se presenta a las Comisiones Regionales del Medio Ambiente. En efecto, la Comisión Regional del Medio Ambiente (Corema) es el ente político administrativo que realiza la gestión ambiental en cada una de las regiones del país. La Corema, es una instancia política administrativa de carácter resolutivo en los temas ambientales, y sus integrantes sólo se reúnen cuando hay proyectos que resolver o algún tema ambiental relevante que abordar' .

La Comisión Nacional del Medio Ambiente en cada una de las regiones es la encargada de administrar el SEIA; además coordina el Comité Operativo de Fiscalización Regional (COFR), que está constituido por todos los servicios públicos con competencia ambiental, o que tengan alguna competencia relevante para el proyecto a fiscalizar ${ }^{10}$. En este sentido, el sistema de fiscalización considerado por el SEIA es eminentemente multi-

yecto y, además, establece cuáles son los permisos ambientales sectoriales aplicables a los proyectos. De igual forma, el Reglamento también señala los procedimientos administrativos que se deben cumplir y los contenidos principales de la participación de la comunidad en la evaluación ambiental. Finalmente, el Reglamento establece que corresponde a los organismos del Estado que participan en el SEIA fiscalizar el permanente cumplimiento de las normas y condiciones con las que se aprobó un determinado proyecto.

${ }^{8}$ La Ley 19300, denominada Ley de Bases Generales del Medio Ambiente, vigente a partir del mes de marzo del año 1994, creó la Comisión Nacional del Medio Ambiente (Conama), organismo que, entre otras responsabilidades, es responsable de coordinar la gestión ambiental nacional.

${ }^{9}$ La Comisión Regional del Medio Ambiente está constituida por las autoridades del gobierno en la región. Específicamente, es presidida por el intendente; ejerce como secretario el director regional de Conama, y la integran además los gobernadores provinciales y secretarios regionales ministeriales. Adicionalmente, participan también consejeros regionales.

${ }^{10}$ Específicamente, los servicios que participan en el Comité de Fiscalización Regional son: Secretaría Regional Ministerial de Salud, Secretaría Regional Ministerial de Vivienda y Urbanismo, Secretaría Regional Ministerial de Agricultura, Secretaría Regional Ministerial de Transportes y Telecomunicaciones, Subsecretaría de Pesca (Subpesca), Superintendencia de Servicios Sanitarios (SISS), Superintendencia de Electricidad y Combustibles (SEC), Corporación Nacional Forestal (CONAF), Servicio Nacional de Pesca (Sernapesca), Consejo de Monumentos Nacionales, Dirección Gobernación del Territorio Marítimo (DGTM), Servicio Agrícola y Ganadero (SAG), Dirección General de Aguas (DGA), Dirección de Vialidad, Servicio Nacional de Geología y Minas (Sernageomin), Servicio Nacional de Turismo (Sernatur). 
sectorial e incluye diversos órganos encargados de verificar el cumplimiento de las normas.

\subsection{Procedimiento de evaluación ambiental en el SEIA}

El procedimiento de evaluación ambiental se inicia con la presentación que debe realizar el titular de un proyecto ante la Conama (ver Figura $\mathrm{N}^{\circ}$ 1). Existen dos formas de presentar el proyecto. Primero, se considera la modalidad denominada Declaración de Impacto Ambiental (DIA), la cual consiste en una descripción general de los principales aspectos del proyec-

FIGURA N ${ }^{\circ}$ 1: $\quad$ ESQUEMA DEL PROCESO DE EVALUACIÓN DE IMPACTO AMBIENTAL

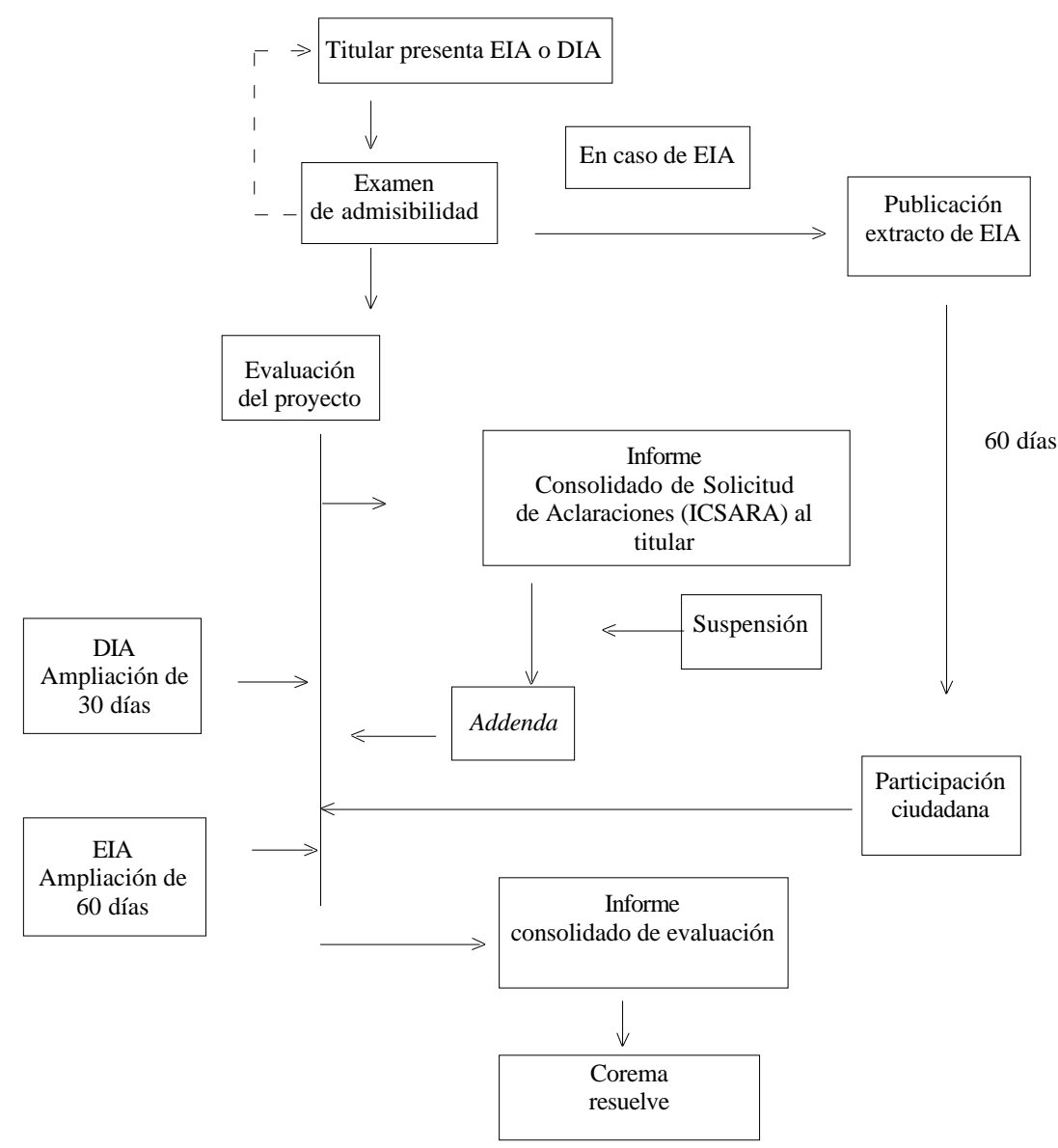

Fuente: Elaboración propia. 
to y sus impactos ambientales. Segundo, existe también la posibilidad de realizar un Estudio de Impacto Ambiental (EIA). En esta modalidad se describen pormenorizadamente todos los aspectos del proyecto, incluyendo, por ejemplo, línea de base del área donde se ejecutará, impactos ambientales generados, plan de contingencias, compromisos voluntarios y medidas de compensación ${ }^{11}$.

En caso que el proyecto se presente bajo la modalidad de Declaración de Impacto Ambiental, el plazo legal para su evaluación y aprobación por parte de la Comisión Regional del Medio Ambiente (Corema) es de 60 días hábiles, ampliables a un máximo de 30 días adicionales por una sola vez. Durante este período, los distintos servicios públicos invitados a revisar el proyecto deben hacer observaciones que en conjunto pasan a constituir el Informe Consolidado de Solicitud de Aclaraciones que el titular del proyecto deberá responder dentro de un plazo legal bajo la forma de un documento, el cual es denominado en el RSEIA como Addenda. Este documento debe contener las aclaraciones solicitadas ${ }^{12}$. Considerando que, luego de revisado el documento Addenda, no existan observaciones adicionales, se elabora un Informe Consolidado de Evaluación, el que resume los aspectos relevantes del proyecto y señala los compromisos ambientales asumidos por el titular. Posteriormente, se presenta este informe a la Corema, instancia que califica ambientalmente el proyecto. No obstante lo anterior, cabe la posibilidad de que en base a la primera revisión de los organismos sectoriales exista consenso en que la Declaración de Impacto Ambiental (DIA) presentada no es suficiente, haciéndose necesario en dicho caso exigir un Estudio de Impacto Ambiental (EIA). En dicho escenario, el coordinador del proyecto - un profesional de Conama - presenta el estado de evaluación del proyecto a la Corema, la que puede resolver en vista de los antecedentes conocidos rechazar la DIA y solicitar un EIA, lo que se le comunica al titular mediante una Resolución Exenta de rechazo del proyecto.

${ }^{11}$ La elección de la modalidad de ingreso al SEIA es un derecho del agente que ejerce como titular o representante del proyecto, la cual está usualmente en función del análisis de los componentes ambientales y las obras que el proyecto contempla y su efecto sobre el medio ambiente. No obstante que existe completa libertad de elección en esta decisión, la experiencia sugiere que ingresan en la modalidad de EIA los proyectos de mayor envergadura, como centrales hidroeléctricas, complejos industriales, y también los proyectos conflictivos, como lo han sido, de acuerdo a la experiencia reciente, los rellenos sanitarios.

${ }^{12}$ Es posible suspender el plazo de evaluación por un período no determinado por la ley, a fin de que el titular disponga de tiempo para responder las observaciones efectuadas a su proyecto. 
Si el proyecto se presenta bajo la forma de un Estudio de Impacto Ambiental (EIA), se realiza el examen de admisibilidad con el propósito de juzgar la pertinencia de acoger el proyecto para su evaluación. En este caso el plazo legal para evaluar el proyecto es de 120 días hábiles, el cual es posible extender, por una sola vez, hasta un máximo de 60 días adicionales. Si el proyecto es admitido e ingresa a evaluación, el titular debe publicar un extracto del EIA en el Diario Oficial y en algún diario de circulación local en la comuna o provincia en que se ejecutará el proyecto. Este requerimiento fue establecido con el propósito de dar inicio al proceso de participación ciudadana, considerado en el Reglamento del SEIA.

El proceso de participación ciudadana bajo la modalidad de presentación de un Estudio de Impacto Ambiental considera un plazo de 60 días hábiles dentro de los cuales la población afectada por la ejecución del proyecto puede hacer observaciones ${ }^{13}$.

En el caso que la Corema resuelve favorablemente la calificación ambiental del proyecto, se redacta la Resolución de Calificación Ambiental (RCA), documento que comprende al Informe Consolidado de Evaluación, en conjunto con otras consideraciones que hayan resultado de la discusión de la Corema al momento de resolver el proyecto. Todo proyecto ingresado al SEIA cuenta con una RCA. En efecto, existe RCA de aprobación, de rechazo, y también de desistimiento. Cuando los proyectos son aprobados, la RCA describe los aspectos principales del proyecto sometido a evaluación, así como también los permisos ambientales sectoriales asociados a él y las obligaciones impuestas por la autoridad a las que el titular del proyecto deberá dar cumplimiento. Si los proyectos son rechazados o desistidos, la RCA indica las causales de tal condición ${ }^{14}$.

${ }^{13}$ El procedimiento para realizar observaciones consiste en presentarlas por escrito a la Dirección Regional de Conama. Ello lo puede hacer cualquier persona natural u organización que acredite su identidad y domicilio, a fin de comprobar si el proyecto la afectará directamente.

${ }^{14}$ Es pertinente mencionar que las RCA que se elaboraban en el inicio del Sistema de Evaluación de Impacto Ambiental se caracterizaban por ser de carácter general, sin proporcionar información técnica precisa respecto de exigencias o aspectos claves del proyecto para ser fiscalizados. Naturalmente, la falta de claridad en las exigencias pudo contribuir, por un lado, a facilitar el incumplimiento, debido al desconocimiento en relación a los alcances de la RCA de parte de los encargados de ejecutar los proyectos. En tanto que, por otro lado, exigencias poco claras también pudieron dificultar la labor fiscalizadora al momento de realizar la fiscalización. Lamentablemente, no tenemos evidencia suficiente para evaluar el impacto que sobre la decisión de cumplimiento ha tenido la técnica de redacción y contenidos de las RCA. No obstante, de acuerdo a nuestra revisión, a medida que se ha ido ganando experiencia en la evaluación y fiscalización de los proyectos, se ha ido paulatinamente mejorando la elaboración de las RCA, lo que probablemente se ha traducido en una mejor comprensión de los alcances de éstas, tanto 
Una vez que el proyecto cuenta con su RCA, puede iniciar su ejecución. Durante dicho período, el proyecto puede ser sometido a fiscalización de parte del Estado. Las actividades de fiscalización consideradas en el SEIA incluyen principalmente visitas a terreno a fin de constatar el cumplimiento de las exigencias ambientales establecidas en la RCA; en caso de encontrar violaciones graves y permanentes, se procede a abrir un expediente sancionatorio. Este procedimiento puede finalizar con la imposición de una multa. En caso de infracciones reiteradas se puede llegar incluso a la revocación de la aprobación o aceptación del proyecto evaluado, sin perjuicio del derecho a ejercer las acciones civiles o penales que sean procedentes $^{15}$.

En particular, son los aspectos indicados en la RCA del proyecto los que se espera orienten la revisión durante las visitas de fiscalización desplegadas por los órganos que participaron en la evaluación ambiental del proyecto.

\section{EL SISTEMA DE EVALUACIÓN DE IMPACTO AMBIENTAL (SEIA) Y LA FISCALIZACIÓN PARA CUMPLIMIENTO DE LA RESOLUCIÓN DE CALIFICACIÓN AMBIENTAL (RCA) EN LA REGIÓN DEL BÍO-BÍO}

El análisis de esta sección considera una breve descripción del funcionamiento del Sistema de Evaluación de Impacto Ambiental en la región del Bío-Bío. Adicionalmente se revisa en detalle el diseño de fiscalización para inducir cumplimiento de la Resolución de Calificación Ambiental del Programa de Fiscalización Ambiental Regional.

\subsection{Funcionamiento del SEIA en la región del Bío-Bío}

En el período 1994-2004 se han sometido a evaluación de impacto ambiental en la región del Bío-Bío un total de 688 proyectos. El monto total

para los fiscalizadores como para los técnicos del titular que ejecutan el proyecto. Ello, muy posiblemente, ha permitido mejorar los niveles de cumplimiento de las exigencias ambientales establecidas. Actualmente, las RCA comprenden por un lado los aspectos generales del proyecto y por otro lado señalan detalladamente los aspectos normados y no normados relevantes a ser fiscalizados, proporcionando una herramienta rápida y precisa para detectar cumplimiento ambiental. Agradecemos a un árbitro anónimo de la revista Estudios Públicos por motivar este punto.

${ }^{15}$ Es interesante mencionar que la imposición de sanciones está basada en un proceso administrativo (expediente sancionatorio). El nivel de la multa (sanción monetaria) es definido por la Corema en base a antecedentes relacionados con el proyecto, es decir, caso a caso. El RSEIA no señala criterios para definir el monto de la sanción monetaria. 
de la inversión representada por dichos proyectos alcanzó a 5.836 millones de dólares.

La Tabla $\mathrm{N}^{\mathrm{o}} 1$ muestra los proyectos ingresados a evaluación, resultados de la evaluación (aprobados, rechazados, desistidos) y modalidad de ingreso (DIA o EIA) al SEIA en la región del Bío-Bío en el período 19942004. Se observa que alrededor del $82 \%$ de los proyectos ingresados son aprobados. Con propósito de comparación, considere que durante el mismo período el número de proyectos ingresados al SEIA a nivel nacional alcanzó a 6.445, de los cuales fueron aprobados 5.246, esto es, la tasa de aprobación alcanzó a 81,4\% (OCDE-CEPAL, 2005).

La mayoría de los proyectos no aprobados corresponde a aquellos que no culminaron el proceso de evaluación (proyectos desistidos) y representa cerca del $12 \%$ de los proyectos ingresados a evaluación. Sólo el 5\% de los proyectos ingresados fue rechazado durante el período.

TABLA N ${ }^{\circ}$ 1: $\quad$ PROYECTOS INGRESADOS A SEIA, RESULTADOS DE EVALUACIÓN Y MODALIDAD DE INGRESO EN LA REGIÓN DEL BÍO-BÍO, PERÍODO 1994-2004

\begin{tabular}{|c|c|c|c|c|c|c|}
\hline Año & $\begin{array}{c}\mathrm{N}^{\mathrm{o}} \text { de } \\
\text { proyectos } \\
\text { ingresados } \\
\text { al SEIA }\end{array}$ & $\begin{array}{c}\mathrm{N}^{\mathrm{o}} \text { de } \\
\text { proyectos } \\
\text { aprobados }^{\mathrm{a}}\end{array}$ & $\begin{array}{l}\mathrm{N}^{\mathrm{o}} \text { de } \\
\text { proyectos } \\
\text { rechazados }\end{array}$ & $\begin{array}{l}\mathrm{N}^{\circ} \text { de } \\
\text { proyectos } \\
\text { desistidos }\end{array}$ & $\begin{array}{c}\mathrm{N}^{\circ} \text { de } \\
\text { proyectos } \\
\text { ingresados } \\
\text { como DIA }\end{array}$ & $\begin{array}{c}\mathrm{N}^{\circ} \text { de } \\
\text { proyectos } \\
\text { ingresados } \\
\text { como EIA }\end{array}$ \\
\hline
\end{tabular}

\begin{tabular}{|c|c|c|c|c|c|c|}
\hline 1994 & 1 & 0 & 0 & 0 & 0 & 1 \\
\hline 1995 & 8 & 3 & 0 & 0 & 0 & 8 \\
\hline 1996 & 5 & 9 & 0 & 0 & 0 & 5 \\
\hline 1997 & 28 & 12 & 1 & 0 & 24 & 4 \\
\hline 1998 & 89 & 51 & 2 & 4 & 85 & 4 \\
\hline 1999 & 119 & 99 & 3 & 3 & 113 & 6 \\
\hline 2000 & 89 & 86 & 1 & 5 & 81 & 8 \\
\hline 2001 & 105 & 104 & 3 & 6 & 100 & 5 \\
\hline 2002 & 107 & 96 & 2 & 19 & 99 & 8 \\
\hline 2003 & 59 & 45 & 0 & 23 & 53 & 6 \\
\hline 2004 & 78 & 62 & 1 & 17 & 76 & 2 \\
\hline Total & 688 & 567 & 13 & 77 & 631 & 57 \\
\hline
\end{tabular}

${ }^{a}$ El número de proyectos ingresados cada año difiere del número de proyectos aprobados en igual período debido a que la decisión sobre proyectos que ingresan a evaluación en un año dado puede ser resuelta durante el mismo período o en años posteriores.

Fuente: Elaboración propia en base a la información proporcionada por el Departamento de Fiscalización de la Dirección Regional de Conama. 
Los proyectos ingresados a evaluación utilizan preferentemente la modalidad de Declaración de Impacto Ambiental (DIA) en lugar de Evaluación de Impacto Ambiental (EIA). Específicamente, durante el período 1994-2004, aproximadamente el 92\% de los proyectos ingresados al SEIA utilizaron la modalidad DIA (ver Tabla $\mathrm{N}^{\circ} 1$, últimas dos columnas). Muy probablemente ello se explica porque, por un lado, los tiempos de evaluación para una DIA son menores que los de una EIA (ver subsección 2.2) y, por otro lado, los costos para elaborar una DIA son menores que aquellos relacionados a una EIA.

\subsection{Fiscalización para el cumplimiento de la RCA en la región del Bío-Bío}

A continuación se presentan dos aspectos fundamentales para la comprensión del Programa de Fiscalización de la región del Bío-Bío. En primer lugar, se describen el procedimiento y estrategia general de fiscalización considerados por el Programa; en segundo lugar, se revisan los resultados de cumplimiento individual posibles que arroja la actividad de visitas de inspección.

\subsubsection{Procedimiento de fiscalización}

Cada año la Dirección Ejecutiva de la Comisión Nacional del Medio Ambiente (Conama) determina el número de proyectos a ser fiscalizados a nivel nacional y dispone los recursos humanos y financieros para ejecutar el Programa de Fiscalización Ambiental Regional (PFAR). En el caso del año 2004 el objetivo definido por la Dirección Nacional fue fiscalizar un total nacional de 390 proyectos. En la región del Bío-Bío se consideró, para el mismo período, una cobertura de fiscalización que alcanzaba a un total de 30 proyectos.

El Comité Operativo de Fiscalización Regional de la Región del BíoBío fue creado durante el año 2001. En dicho período se estableció un procedimiento formal para el desarrollo de la actividad de fiscalización. Este procedimiento se describe a continuación. Desde el nivel local regional, y luego de conocidos los objetivos de cobertura de fiscalización definidos por la Dirección Nacional y al inicio del año calendario respectivo, se solicita a cada uno de los integrantes del Comité Operativo de Fiscalización Regional que señale, a partir del universo de proyectos aprobados, un listado de a lo menos diez proyectos que considere de su interés para ser sujetos de fiscalización durante el referido período ${ }^{16}$. En función de las recomenda-

${ }^{16}$ De acuerdo a lo indicado en la sección 2, el Comité de Fiscalización Regional está integrado por todos los servicios públicos con competencia ambiental. 
ciones individuales de parte de los integrantes del Comité Operativo de Fiscalización Regional, la Conama de la región del Bío-Bío prepara un listado consolidado de los proyectos señalados y procede a su selección de acuerdo con la siguiente clasificación: proyectos conflictivos (que corresponden a aquellos proyectos que durante su evaluación motivaron reclamos de personas naturales $\mathrm{y} / \mathrm{u}$ organizaciones sociales), proyectos que se han fiscalizado y que han presentado cumplimiento parcial, proyectos que han presentado incumplimiento total, proyectos aprobados en años anteriores que no han sido fiscalizados y proyectos que han sido recientemente aprobados. Posteriormente, y considerando el total de proyectos previamente seleccionados y clasificados, se procede a consultar a los evaluadores de Conama que coordinaron la revisión de dichos proyectos. Finalmente, son precisamente los proyectos sugeridos por dichos profesionales los que serán visitados para inspección, como parte de la actividad de fiscalización.

Las visitas de fiscalización a proyectos aprobados bajo el SEIA se realizan por invitación de la Conama. Dicha invitación es extendida a los servicios que evaluaron ambientalmente los proyectos de interés. Los elementos centrales para realizar la fiscalización incluyen la RCA, informes sectoriales y/o denuncias por parte de la comunidad. La fiscalización que se realiza considera una revisión de los antecedentes relevantes establecidos en la RCA, los cuales se contrastan con el estado de ejecución del proyecto. En la práctica sólo se consideran aspectos ambientales señalados en la RCA que son de fácil verificación. En particular, no se toman muestras de agua, ni tampoco de residuos líquidos, u otros tipos de efluentes o emisiones, para propósitos de realizar análisis cuantitativos. Lo anterior no es posible, pues no se cuenta con el equipamiento requerido para ello. La fiscalización consiste, por un lado, en inspección visual de la ejecución de las principales obras en las etapas de construcción y operación; por otro lado, la fiscalización considera también la realización de una entrevista con los encargados de la ejecución del proyecto. Adicionalmente se revisan las bitácoras de operación del proyecto y de los monitoreos realizados, si los hubiera ${ }^{17}$.

${ }^{17}$ El carácter de inspección visual de las visitas de fiscalización constituye una limitación importante de los esfuerzos de fiscalización en la región. En efecto, dado el tipo de inspección que se realiza, no es posible revisar el estatus de cumplimiento en relación, por ejemplo, con el límite de emisiones o efluentes, los cuales en algunos casos están establecidos en la Resolución de Calificación Ambiental. En este sentido la tecnología de inspección muestra limitaciones y el estatus de cumplimiento se define en función de la inspección visual. 


\subsubsection{Cumplimiento y sanciones en el Programa de Fiscalización Ambiental Regional}

Considerando aspectos de procedimiento establecidos por el PFAR, nuestra revisión de las fichas de fiscalización, y comentarios de parte del encargado regional de la ejecución del PFAR de la región del Bío-Bío, los que fueron obtenidos a través de comunicación personal de uno de los autores de este trabajo, definimos tres estatus de cumplimiento, éstos son:

A. Cumplimiento total: Se encuentran en esta condición aquellos proyectos que se ejecutan y operan de acuerdo con todas las exigencias establecidas en la RCA.

B. Cumplimiento parcial: Se define como aquel en que se constata que el titular ha ejecutado el proyecto en sus distintas etapas considerando las exigencias ambientales de la RCA; sin embargo, se han omitido ciertos aspectos formales e importantes, lo que muestra que no hubo un cumplimiento total. En este caso es posible encontrar las siguientes situaciones de cumplimiento específicas:

Cumplimiento alto de la RCA: Están en dicha situación proyectos que exhiben aspectos menores deficientes en relación con lo establecido en la RCA o demás normas de ejecución, que son fácilmente subsanables por el ejecutor. Se incluyen aquí, por ejemplo, proyectos que no hayan dado aviso por escrito a Conama del inicio de obras, o que no hayan enviado el o los Permisos Ambientales Sectoriales (PAS) otorgados a fin de informar a la autoridad, o que hayan omitido la ejecución de algún compromiso voluntario asumido en el proceso de evaluación del proyecto ${ }^{18}$.

Cumplimiento medio de la RCA: Se considera en estado de cumplimiento medio a aquellos proyectos que durante la fiscalización se encuentran operando sin la totalidad de los PAS exigidos por la RCA, es decir, que no cuentan con la otorgación de ellos, y por ende no han dado cuenta formal de ellos a la autoridad.

Cumplimiento bajo de la RCA: En esta categoría se encuentran los proyectos que durante la visita de fiscalización se encuentran funcionando sin contar con ninguno de los PAS asociados al proyecto, o que hayan sido detectados como infractores permanentes en las visitas de fiscalización anteriores.

${ }^{18}$ Los PAS están referidos a las soluciones sanitarias de: tratamiento de aguas servidas, residuos industriales líquidos, disposición de residuos sólidos, sistema de purificación de agua potable, u otro permiso sectorial en incumplimiento. 
C. Incumplimiento total de la RCA: Se consideran en esta situación aquellos proyectos ejecutados por el titular sin considerar las exigencias ambientales establecidas en la RCA. La ejecución no concuerda con las especificaciones del proyecto evaluado y aprobado, y por ende se ha ejecutado un proyecto distinto del que fue aprobado por la Corema. Se entiende, en este caso, que el proyecto no cumple con las exigencias ambientales establecidas por la Corema.

Realizadas las visitas de fiscalización y evaluados los antecedentes recopilados, los proyectos fiscalizados son clasificados en alguno de los estatus o condiciones de cumplimiento previamente revisados. En función de dicha situación individual, se contemplan diversas acciones adicionales en el ámbito del PFAR. Específicamente, las referidas acciones asociadas a los resultados de cada visita de fiscalización para cada situación de (in)cumplimiento son las siguientes:

En el caso de cumplimiento total (Caso A), se deja constancia de tal condición en el acta de fiscalización que se le envía al titular del proyecto. Adicionalmente se le reconoce oficialmente su condición y se le estimula a continuar con sus procedimientos de manera de velar por la mantención de los compromisos cumplidos. Finalmente, se le comunica que el proyecto continúa siendo considerado en el universo de proyectos fiscalizables en períodos futuros.

En el caso de cumplimiento parcial (Caso B), se deja constancia en el acta de fiscalización respecto de los aspectos de la RCA que han sido encontrados en (in)cumplimiento parcial. En cada caso específico se procede como sigue. Si el proyecto resulta en cumplimiento parcial alto, se envía al titular del proyecto una carta que contiene una amonestación escrita. En dicha carta se comunica al titular del proyecto la(s) falta(s) en que ha incurrido y se le solicita remitir los antecedentes que informen cómo han sido ésta(s) subsanada(s) dentro de un breve plazo. En caso de cumplimiento medio de la RCA, se presenta la situación ante la Corema para la toma de conocimiento y resolución. Adicionalmente, se envía una carta al titular del proyecto con una amonestación escrita. En dicha comunicación se identifican: la(s) falta(s) en que incurrió y se establece un plazo para remediarla(s). Se informa además que se le visitará nuevamente. Finalmente, en caso de cumplimiento parcial bajo de la RCA, se presenta la situación ante la Corema para la toma de conocimiento y resolución, la que resuelve respecto a amonestar por escrito al titular del proyecto. En dicha amonestación se le comunica al titular la gravedad de su proceder y se le reitera la obligación que éste tiene de subsanar sus faltas, indicándole que en caso contrario se 
procederá a la apertura de un expediente sancionatorio, a aplicarle una multa, e incluso, dependiendo del caso, a la revocación de la RCA.

Los proyectos fiscalizados pueden también exhibir la condición de incumplimiento total de la RCA (Caso C). Si así ocurriera, se procede a presentar la situación ante la Corema para la toma de conocimiento y resolución. Esta instancia puede resolver abrir un expediente sancionatorio y/o multar directamente al infractor; además de exigir subsanar la situación de violación a la brevedad. El expediente sancionatorio es un conjunto de información detallada referida al proyecto encontrado en violación, que incluye todos los aspectos ambientales del proyecto, esto es, los informes sectoriales emitidos por los revisores que evaluaron el proyecto, la(s) Addenda(s) emitida(s) por el titular para responder a las observaciones realizadas al proyecto, la RCA, las fichas de fiscalización y otros antecedentes si los hubiere. Si se abre el expediente sancionatorio, se le da la posibilidad al infractor de enmendar su falta dentro de un plazo determinado, período en que se sigue fiscalizando al infractor a fin de ir revisando su accionar y su estado de cumplimiento. Posteriormente, la Corema da inicio a la evaluación del expediente sancionatorio. Este procedimiento puede concluir con la imposición de una sanción monetaria (ver sección 2.2). Finalmente, en el caso extremo de que el titular no acoja las demandas de la Corema se puede llegar a la revocación de la RCA, con lo cual se cierra el expediente y se prohíbe la operación del proyecto. Adicionalmente, todo el proceso es de conocimiento público, se informa a la comunidad mediante la prensa ordinaria y la publicación en el Diario Oficial.

\section{RESULTADOS DE CUMPLIMIENTO DE RCA EN LA REGIÓN DEL BÍO-BÍO}

Esta sección presenta los resultados del análisis de la actividad de fiscalización realizada por el PFAR en la región del Bío-Bío, en términos de cumplimiento de las exigencias ambientales de los proyectos fiscalizados durante el período $1999-2004^{19}$.

\subsection{Datos}

La base de datos utilizada está formada por observaciones de inspecciones que se han realizado en el marco de las actividades del Programa de Fiscalización Regional (PFAR) durante el período de estudio. La fiscaliza-

${ }^{19}$ El PFAR en la región del Bío-Bío se inició durante el año 1999 y su ejecución tuvo una interrupción en el año 2000. El Programa fue ejecutado nuevamente el año 2001 y se ha mantenido activo desde entonces. 
ción de los proyectos se realizó en los años 1999, 2001, 2002, 2003 y el primer semestre del año 2004. La base de datos comprende los siguientes campos: identificación del proyecto sometido a visita de inspección cada año, mes en que se realizó la fiscalización, número de visitas de fiscalización previas, nivel de exigencia ambiental, número de plantas del proyecto fiscalizado, duración del proyecto (temporal, permanente), localización del proyecto, población de la comuna en que se localiza el proyecto (población total, población urbana y población rural), monto de inversión asociado al proyecto, nivel de ocupación de mano de obra, modalidad de presentación al SEIA (EIA o DIA), tipos de proyecto según reglamento del SEIA, indicador de conflictividad del proyecto visitado, duración del período de evaluación del proyecto, antigüedad del proyecto, y el estatus de cumplimiento del proyecto de acuerdo con la descripción de la sección $3^{20}$.

\subsection{Proyectos fiscalizados en la región del Bío-Bío en el período de estudio}

Una síntesis de la actividad de fiscalización de proyectos aprobados bajo el SEIA en la región del Bío-Bío durante el período 1999-2004 se presenta en la Tabla $\mathrm{N}^{\circ}$ 2. Se observa allí el número de RCA aprobadas por año, el universo de proyectos fiscalizables, el número de visitas realizadas por año y agregadas, el número de proyectos fiscalizados, el número de proyectos nuevos fiscalizados cada año, el número de proyectos eliminados y el número de proyectos que se repiten en las fiscalizaciones realizadas.

Es importante notar que el número de visitas de inspección no corresponde al número de proyectos fiscalizados, debido a que existen proyectos que fueron visitados más de una vez. Adicionalmente, de acuerdo con lo informado por los encargados de coordinar la fiscalización en la región del Bío-Bío, en base a criterios previamente definidos, se considera que una visita es una fiscalización, independientemente de que sea el mismo proyecto que se visite a lo largo del tiempo. Así, por ejemplo, se observa en la Tabla $N^{\circ} 2$ que en el año 2001 fueron desarrolladas 27 visitas de inspección, correspondientes a 21 proyectos fiscalizados, de los cuales 18 se visitaban por primera vez y 3 se inspeccionaban por segunda vez; mientras que durante el año 2003 se efectuaron 59 visitas que correspondieron a 56 proyectos fiscalizados, de los cuales 40 eran visitados por primera vez y 16 ya habían sido visitados anteriormente.

${ }^{20}$ Con el propósito de realizar el análisis estadístico, cada visita de fiscalización es considerada de manera independiente de cualquier otra visita. De esta manera, más de una visita al mismo proyecto es considerada como fiscalización adicional. 
TABLA N 2 $^{2} \quad$ ACTIVIDAD DE FISCALIZACIÓN DE RCA EN LA REGIÓN DEL BÍO-BIO: 1994-2004

\begin{tabular}{lrrrrrrr}
\hline Año & $\mathrm{RCA}^{\mathrm{a}}$ & $\mathrm{PF}^{\mathrm{b}}$ & $\mathrm{VF}^{\mathrm{c}}$ & $\mathrm{PF}^{\mathrm{d}}$ & $\mathrm{PNF}^{\mathrm{e}}$ & $\mathrm{PE}^{\mathrm{f}}$ & $\mathrm{PR}^{\mathrm{g}}$ \\
\hline $1994-1998$ & 75 & 75 & 0 & 0 & 0 & 0 & 0 \\
1999 & 99 & 174 & 15 & 15 & 15 & 0 & 0 \\
2000 & 86 & 260 & 0 & 0 & 0 & 0 & 0 \\
2001 & 104 & 364 & 27 & 21 & 18 & 12 & 3 \\
2002 & 96 & 460 & 80 & 56 & 47 & 15 & 9 \\
2003 & 45 & 505 & 59 & 56 & 40 & 40 & 16 \\
$2004^{\mathrm{h}}$ & 62 & 567 & 23 & 22 & 11 & 43 & 11 \\
Total & 567 & & 204 & 170 & 131 & 110 & 39 \\
\hline
\end{tabular}

a Considera el número total de RCA aprobadas en el período 1994-primer semestre del año 2004.

${ }^{\mathrm{b}}$ Es el universo de proyectos fiscalizables, incluye los proyectos con RCA aprobados hasta el año inmediatamente anterior.

${ }^{\mathrm{c}}$ Total visitas de fiscalización realizadas cada año.

d Número de proyectos fiscalizados, difiere del total de visitas debido a que algunos proyectos se fiscalizan más de una vez cada año. vez fiscalizados.

e Proyectos nuevos fiscalizados, incluye a los proyectos que son por primera

${ }^{\mathrm{f}}$ Proyectos eliminados, es decir aquellos que no se fiscalizan en el período.

g Proyectos repetidos, esto es, proyectos que han sido fiscalizados más de una vez en el período de estudio.

${ }^{\mathrm{h}}$ Incluye sólo información para el primer semestre del año.

Fuente: Elaboración propia en base a información del PFAR, región del Bío-Bío.

Se observa también en la Tabla Nº 2 que en el año 1999 se fiscalizó el $12,7 \%$ de los proyectos respecto del universo de proyectos fiscalizables. La mayor cobertura de fiscalización se logró durante el año 2002, período en el cual se fiscalizó el 14,4\% del universo de proyectos con RCA aprobada. Durante los siguientes períodos la tasa de cobertura de visitas de inspección se redujo, alcanzando a 11,9\% durante el año 2003, y poco más de 4\% durante el primer semestre del año 2004. No obstante que no disponemos de patrones claros de comparación, una cobertura de fiscalización que alcanza a alrededor del $10 \%$ del universo fiscalizable al año no parece excesivamente reducida.

Adicionalmente, nuestro análisis indica también que los proyectos nuevos fiscalizados alcanzan un alto porcentaje, no así los proyectos que han sido visitados anteriormente. En efecto, por ejemplo, para los años 
2001, 2002 y 2003, los proyectos nuevos representan en promedio el 80\% de los proyectos fiscalizados durante esos tres años (ver Tabla № 2).

Una característica relevante de la fiscalización de proyectos aprobados bajo el SEIA lo constituye la participación de diversos servicios públicos; esto es, existe más de una agencia u órgano fiscalizador sectorial. La Tabla No 3 muestra el número promedio de servicios públicos fiscalizadores que participaron en las visitas de fiscalización realizadas cada año durante el período de estudio, así como también la desviación estándar asociada. Se aprecia que el número promedio de servicios que participan en las visitas de fiscalización fluctúa entre 3 y 4 en cada año del período considerado, con una desviación estándar que bordea la unidad. Estas cifras confirman el carácter multisectorial de las visitas de fiscalización para inducir cumplimiento de la RCA, de acuerdo al diseño institucional vigente.

Considerando la totalidad de las 204 visitas de fiscalización realizadas en el período comprendido por este estudio, se logró fiscalizar 14 tipos de proyectos de acuerdo a la clasificación establecida en el Reglamento del SEIA. La distribución de proyectos fiscalizados por tipo se presenta en la Tabla $N^{\circ} 4$.

La tipología de proyectos más visitados corresponde a aquellos proyectos de saneamiento ambiental, que concentran 81 de las 204 visitas de fiscalización realizadas y representan el 39,7\% del total. Los siguientes tipos de proyectos más visitados son aquellos referidos a recursos hidrobiológicos con 20 visitas (9,80\%), proyectos inmobiliarios con 19 visitas $(9,31 \%)$, y proyectos mineros con 18 visitas $(8,82 \%)$.

TABLA N ${ }^{\circ}$ 3: $\quad$ NÚMERO PROMEDIO DE SERVICIOS QUE PARTICIPAN EN LAS VISITAS DE FISCALIZACIÓN EN LA REGIÓN DEL BÍO-BÍO, 1999-2004

\begin{tabular}{lcc}
\hline Año & $\begin{array}{c}\text { Número promedio de servicios } \\
\text { públicos que participan } \\
\text { en las visitas fiscalizadoras }{ }^{\mathrm{a}}\end{array}$ & Desviación estándar \\
\hline 1999 & 3,9 & 0,998 \\
2001 & 2,9 & 0,587 \\
2002 & 2,9 & 0,997 \\
2003 & 3,1 & 1,029 \\
Primer semestre de 2004 & 3,5 & 1,440 \\
Total & 3,3 & 1,010 \\
\hline
\end{tabular}

${ }^{\text {a }}$ Considera el total de los servicios participantes dividido por el número de visitas fiscalizadoras realizadas en cada año.

Fuente: Elaboración propia. 
TABLA N ${ }^{\circ}$ 4: $\quad$ TIPOS DE PROYECTOS FISCALIZADOS EN LA REGIÓN DEL BÍO-BÍO SEGÚN CLASIFICACIÓN DEL RSEIA, 1999-2004

\begin{tabular}{llccccc}
\hline Tipo de proyectos & 1999 & 2001 & 2002 & 2003 & $1^{\text {er }}$ semestre & $\begin{array}{c}\text { Total } \\
\text { visitas (\%) }\end{array}$
\end{tabular}

\begin{tabular}{|c|c|c|c|c|c|c|c|}
\hline Extracciones de fangos & & & & & 1 & 1 & $(0,49 \%)$ \\
\hline Líneas de transmisión eléctricas & & & 1 & 1 & & 2 & $(0,98 \%)$ \\
\hline Centrales hidroeléctricas & 2 & 2 & 1 & 1 & & 6 & $(2,94 \%)$ \\
\hline $\begin{array}{l}\text { Equipamiento: estacionamientos, } \\
\text { estaciones de servicios, } \\
\text { terminal de buses, carreteras, etc. }\end{array}$ & & & 12 & 3 & & 15 & $(7,35 \%)$ \\
\hline Portuarios & & & 1 & & 2 & 3 & $(1,47 \%)$ \\
\hline Inmobiliarios & & 2 & 5 & 9 & 3 & 19 & $(9,31 \%)$ \\
\hline Cambios de combustibles & & & 4 & 1 & & 5 & $(2,45 \%)$ \\
\hline Mineros & 5 & 3 & 8 & 2 & & 18 & $(8,82 \%)$ \\
\hline Gasoductos & & 2 & & 2 & & 4 & $(1,96 \%)$ \\
\hline Plantas químicas & & 1 & 3 & 6 & 1 & 11 & $(5,39 \%)$ \\
\hline Forestales & 3 & 4 & 3 & 4 & 3 & 17 & $(8,33 \%)$ \\
\hline Acuícolas & 2 & 1 & 7 & 9 & 1 & 20 & $(9,80 \%)$ \\
\hline Transporte terrestre & & & 1 & 1 & & 2 & $(0,98 \%)$ \\
\hline $\begin{array}{l}\text { Saneamiento ambiental } \\
\text { (plantas de tratamiento, } \\
\text { rellenos sanitarios, etc.) }\end{array}$ & 3 & 12 & 34 & 20 & 12 & 81 & $(39,7 \%)$ \\
\hline Total & 15 & 27 & 80 & 59 & 23 & & 204 \\
\hline
\end{tabular}

Fuente: Elaboración propia en base a información del PFAR, región del Bío-Bío.

\subsection{Cumplimiento ambiental en la región del Bío-Bío}

Considerando nuestra revisión de los resultados posibles de una visita de fiscalización de un proyecto aprobado (sección 3.2), revisamos a continuación los resultados de cumplimiento observado a partir de las visitas de inspección bajo el PFAR en el período considerado.

\subsubsection{Cumplimiento de RCA en la región del Bío-Bío: 1999-2004}

La Tabla $\mathrm{N}^{\circ} 5$ muestra el estado de cumplimiento agregado, por año analizado, para los proyectos aprobados bajo el SEIA y que fueron fiscalizados por el PFAR de la región del Bío-Bío en el período 1999-2004.

Nuestro análisis indica que de las 204 visitas de fiscalización, en un total de 102 (50\%) se cumplen a cabalidad con las exigencias ambientales establecidas en la RCA en la región del Bío-Bío durante el período analiza- 
Estatus de cumplimiento 19992001 2002

$20032004^{\text {a }} \begin{gathered}\text { Total } \\ \text { período }\end{gathered}$

\begin{tabular}{lrrrrrr} 
Cumplimiento total & 13 & 13 & 43 & 27 & 6 & 102 \\
Cumplimiento parcial & 2 & 12 & 34 & 30 & 16 & 94 \\
$\quad$ Cumplimiento bajo & 0 & 1 & 15 & 11 & 5 & 32 \\
$\quad$ Cumplimiento medio & 0 & 3 & 9 & 7 & 6 & 25 \\
$\quad$ Cumplimiento alto & 2 & 8 & 10 & 12 & 5 & 37 \\
Incumplimiento total & 0 & 2 & 3 & 2 & 1 & 8 \\
Total visitas de fiscalización & 15 & 27 & 80 & 59 & 23 & 204 \\
\hline
\end{tabular}

${ }^{\text {a }}$ Considera sólo el primer semestre del año.

Fuente: Elaboración propia.

do. Sin embargo, la incidencia de perfecto cumplimiento con la RCA muestra variaciones importantes entre los años considerados. En el año 1999, 13 de los proyectos fiscalizados $(86,7 \%$ del total de visitas de fiscalización del período) exhiben cumplimiento total, en tanto que en el año 2001 se observa que sólo en 13 de las visitas de fiscalización se observa cumplimiento total (48,1\% de las visitas de fiscalización del período). Durante el año 2002 la tasa de cumplimiento total se eleva a 53,8\%; para el año 2003 se observa en dicha situación en el 45,8\% de las visitas de fiscalización. Finalmente, para el primer semestre de 2004 se observó que sólo un 26\% de las visitas de fiscalización arroja como resultado cumplimiento total de las exigencias ambientales de la RCA (ver Tabla $\mathrm{N}^{\circ}$ 5).

En el mismo período considerado existe un total de 94 visitas de fiscalización en las que se detectó cumplimiento parcial, lo que representa el 46,1\% del total de las visitas de fiscalización. De acuerdo con la clasificación provista en la sección 3, el referido total se desagrega como sigue: cumplimiento bajo en 32 visitas, lo cual corresponde al 15,7\% de las visitas realizadas en el período de estudio; cumplimiento medio en 25 visitas, representando un $12,3 \%$ de las visitas realizadas en ese período, y con cumplimiento alto un total de 37 visitas, equivalente a $18,1 \%$ de las visitas realizadas en el período.

Finalmente, existe un total de 8 visitas en las que se detectó incumplimiento total, lo que representa el 3,9\% del total de visitas del período. Por lo anterior, se aprecia que las violaciones a las exigencias ambientales de la 
RCA se presentan principalmente en la categoría de cumplimiento parcial en sus distintas subcategorías.

Adicionalmente, decidimos examinar los resultados de cumplimiento de la RCA según tipo de proyecto de acuerdo a la clasificación considerada en el RSEIA. Un detalle de resultados de cumplimiento por tipo de proyecto se presenta en la Tabla $\mathrm{N}^{\circ}$ 6. Se observa allí que del total de tipos de proyectos considerados, las mayores incidencias de infracciones a la RCA se concentran en aquellos proyectos de saneamiento ambiental, proyectos acuícolas, mineros y forestales. Específicamente, para el año 2001 de las 12 visitas realizadas a este tipo de proyectos se observó que en cinco de ellas se encontró incumplimiento (bajo, medio, alto); para el año 2002, de las 34 visitas realizadas a este tipo de proyectos se tiene que en 17 de ellas se encontró algún grado de incumplimiento; esto es, la tasa de incumplimiento en este tipo de proyectos alcanzaba a 50\%. Finalmente, de las 20 visitas realizadas a este tipo de proyectos durante el año 2003, en 11 de ellas se encontró algún tipo de incumplimiento; uno de los proyectos fiscalizados exhibía incumplimiento total.

En el caso de proyectos acuícolas se observan infracciones durante los años 2002 y 2003. Durante el año 2002, de las siete visitas realizadas a este tipo de proyectos, en cuatro de ellas se encontró algún grado de incumplimiento; en tanto que para el año 2003 de las mueve visitas realizadas, en seis de ellas se encontró incumplimiento (ver Tabla No 6).

\subsubsection{Características de los proyectos infractores y acciones para inducir su cumplimiento}

\section{a) Esfuerzo de fiscalización y resultados de cumplimiento por comunas}

El análisis de los resultados de cumplimiento de la RCA por comuna proporciona información respecto del esfuerzo de fiscalización desplegado por los organismos competentes, así como del nivel de cumplimiento ambiental que genera dicho esfuerzo en relación con los proyectos que han sido aprobados ambientalmente durante el período estudiado.

El universo de proyectos fiscalizados durante el período 1999-2004 en la región del Bío-Bío está distribuido en 34 comunas de la región. Las comunas que recibieron la mayor cantidad de visitas de fiscalización son Talcahuano, Coronel, Los Ángeles y Chillán (ver Tabla N 7). Estas comunas concentraron 91 de las 204 visitas de fiscalización desarrolladas durante el período estudiado, lo que corresponden al 45,1\%. 


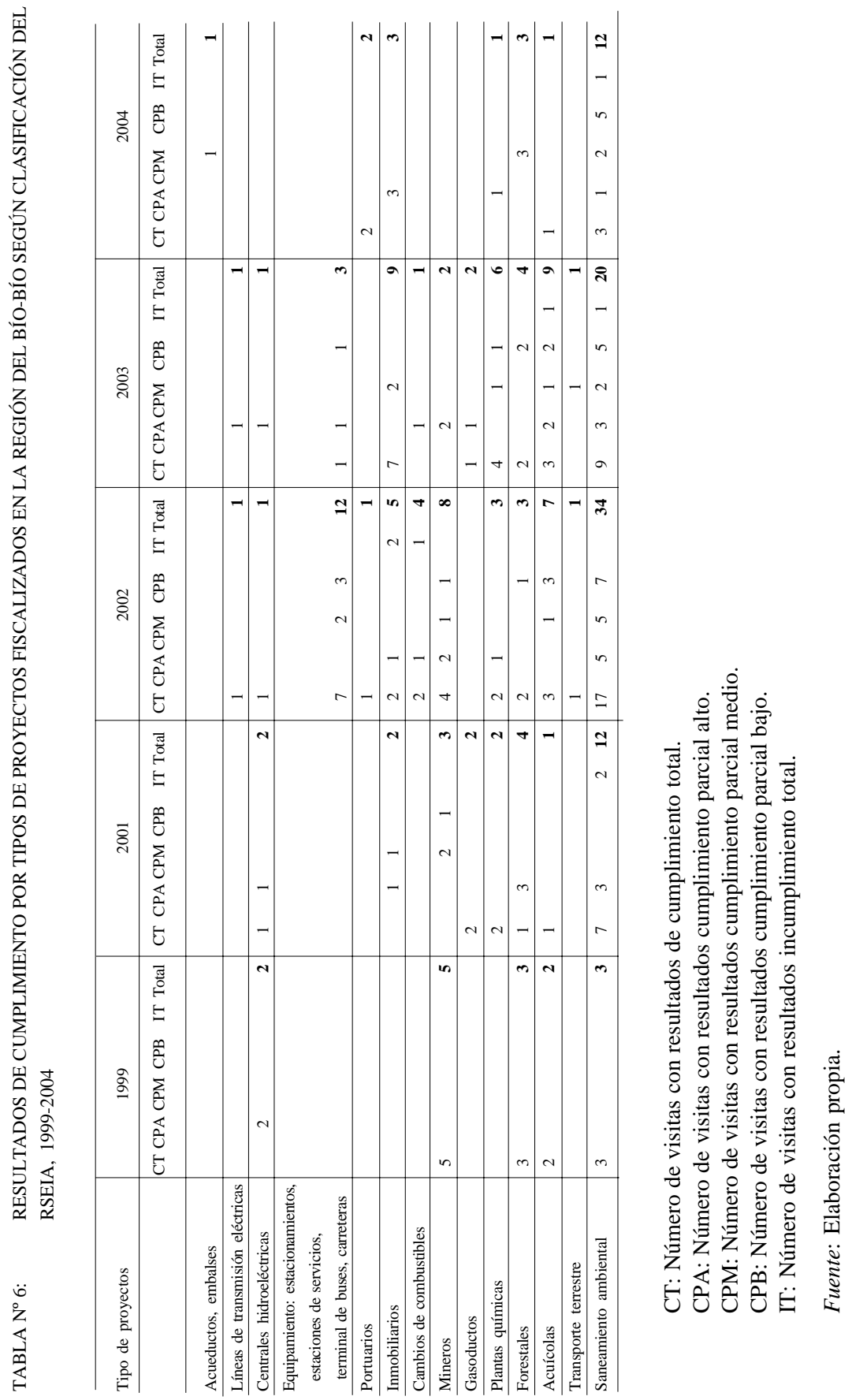


TABLA N 7: $\quad$ VISITAS DE FISCALIZACIÓN POR COMUNAS EN LA REGIÓN DEL BÍO-BÍO: 19992004

\begin{tabular}{lrrrrrrr}
\hline Comunas & 1999 & 2001 & 2002 & 2003 & $2004^{\mathrm{a}}$ & \multicolumn{2}{l}{ Total } \\
\hline Talcahuano & 4 & 1 & 8 & 8 & 0 & 21 & $(10,29 \%)$ \\
Coronel & 2 & 4 & 4 & 10 & 2 & 22 & $(10,78 \%)$ \\
Los Ángeles & 3 & 2 & 7 & 10 & 1 & 23 & $(11,27 \%)$ \\
Chillán & 0 & 1 & 19 & 3 & 2 & 25 & $(12,25 \%)$ \\
Resto comunas & 6 & 19 & 42 & 28 & 18 & 113 & $(55,41 \%)$ \\
Total & 15 & 27 & 80 & 59 & 23 & 204 & $(100 \%)$ \\
\hline
\end{tabular}

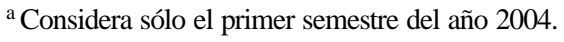

Fuente: Elaboración propia.

La comuna de Talcahuano, predominantemente industrial y de gran incidencia en la producción manufacturera de la región del Bío-Bío, concentra el 10,3\% de las visitas de fiscalización realizadas durante el período 19992004, ocupando el cuarto lugar de las comunas con mayor cantidad de visitas fiscalizadoras.

En la Tabla $\mathrm{N}^{\circ} 8$ se presenta el detalle de cumplimiento ambiental de los proyectos visitados. Durante el período considerado, los proyectos fiscalizados corresponden a cinco tipos, según la clasificación establecida en

TABLA N ${ }^{\circ}$ 8: $\quad$ CUMPLIMIENTO AMBIENTAL EN COMUNA DE TALCAHUANO SEGÚN TIPO DE PROYECTO: 1999-2004

\begin{tabular}{lccccrr}
\hline Tipos de proyectos & $\mathrm{CT}^{\mathrm{a}}$ & $\mathrm{CPA}^{\mathrm{b}}$ & $\mathrm{CPM}^{\mathrm{c}}$ & $\mathrm{CPB}^{\mathrm{d}}$ & $\mathrm{IT}^{\mathrm{e}}$ & Total \\
\hline Cambios de combustibles & & 1 & & & 1 \\
\hline Mineros & 3 & & & & 3 \\
\hline Gasoductos & 2 & & & & 2 \\
\hline $\begin{array}{l}\text { Plantas químicas } \\
\begin{array}{l}\text { Saneamiento ambiental } \\
\text { (plantas de tratamiento, } \\
\text { rellenos sanitarios, etc.) }\end{array}\end{array}$ & 9 & 1 & 2 & & 3 \\
\hline Total & 17 & 2 & 2 & 0 & 0 & 21 \\
\hline
\end{tabular}

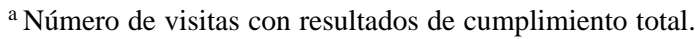

${ }^{\mathrm{b}}$ Número de visitas con resultados de cumplimiento parcial alto.

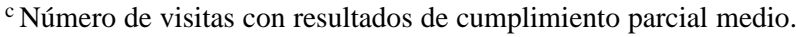

d Número de visitas con resultados de cumplimiento parcial bajo.

e Número de visitas con resultados de incumplimiento total.

Fuente: Elaboración propia. 
TABLA No 9:

CUMPLIMIENTO AMBIENTAL EN COMUNA DE CORONEL SEGÚN TIPO DE PROYECTO: 1999-2004

\begin{tabular}{|c|c|c|c|c|c|c|}
\hline Tipos de proyectos & $\mathrm{CT}^{\mathrm{a}}$ & $\mathrm{CPA}^{\mathrm{b}}$ & $\mathrm{CPM}^{\mathrm{c}}$ & $\mathrm{CPB}^{\mathrm{d}}$ & $\mathrm{IT}^{\mathrm{e}}$ & Total \\
\hline $\begin{array}{l}\text { Equipamiento: estacionamientos, } \\
\text { estaciones de servicios, } \\
\text { terminal de buses, carreteras, etc. }\end{array}$ & & & 1 & 2 & & 3 \\
\hline Gasoductos & & 1 & & & & 1 \\
\hline Plantas químicas & & & & 1 & & 1 \\
\hline Forestales & 1 & 2 & & & & 3 \\
\hline Acuícolas & 4 & & & & & 4 \\
\hline Transporte terrestre & 1 & & 1 & & & 2 \\
\hline $\begin{array}{l}\text { Saneamiento ambiental } \\
\text { (plantas de tratamiento, } \\
\text { rellenos sanitarios, etc.) }\end{array}$ & 1 & 1 & 1 & 3 & 2 & 8 \\
\hline Total & 7 & 4 & 3 & 6 & 2 & 22 \\
\hline
\end{tabular}

${ }^{a}$ Número de visitas con resultados de cumplimiento total.

b Número de visitas con resultados de cumplimiento parcial alto.

${ }^{\mathrm{c}}$ Número de visitas con resultados de cumplimiento parcial medio.

${ }^{\mathrm{d}}$ Número de visitas con resultados de cumplimiento parcial bajo.

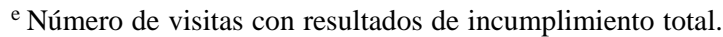

Fuente: Elaboración propia.

el RSEIA. Se observa allí una alta tasa de cumplimiento; de los 21 proyectos fiscalizados, 17 de ellos estaban en tal situación (81\%). Los tipos de incumplimiento detectados no son severos y corresponden principalmente a proyectos de saneamiento ambiental.

La comuna de Coronel concentra el 10,8\% de las visitas de fiscalización realizadas durante el período 1999-2004, ocupando el tercer lugar de las comunas con mayor cantidad de visitas de fiscalización. En la Tabla $\mathrm{N}^{\circ} 9$ se presenta el detalle de cumplimiento ambiental de los proyectos visitados. En esta comuna se presentan siete tipos de proyectos fiscalizados, la mayoría de ellos corresponde a saneamiento ambiental. En el período analizado se detectaron todos los estatus de cumplimiento ambiental; específicamente esta comuna presenta el 31,8\% de cumplimiento total, $18,2 \%$ de cumplimiento parcial alto, $13,6 \%$ de cumplimiento parcial medio, $27,3 \%$ de cumplimiento parcial bajo y 9,1 de incumplimiento total. Este último caso corresponde a los dos proyectos de saneamiento ambiental con visitas de fiscalización durante el período. 
Un análisis desagregado de resultados de fiscalización según tipo de proyectos fiscalizados durante el período 1999-2004 para las comunas de Los Ángeles y Chillán se presenta en el Anexo 1, Tabla A-1 y Tabla A-2, respectivamente.

\section{b) Perfil de los infractores}

Consideramos a continuación un perfil de los proyectos sorprendidos infringiendo la RCA. La caracterización que se realiza se concentra en aquellos proyectos cuya visita de fiscalización arrojó cumplimiento bajo (CB) e incumplimiento total (IT). Nuestro análisis considera las siguientes características: sector al que pertenecen los proyectos, comuna en que se localizan, y un indicador de reincidencia de infracciones de parte de los mismos.

De acuerdo a nuestro análisis, es posible señalar que los infractores se concentran mayoritariamente en los proyectos de saneamiento ambiental; 12 de los 18 proyectos con incumplimiento severo de la RCA corresponden a esta categoría. El resto de proyectos con incumplimiento parcial alto y total se distribuye entre aquellos de equipamiento, inmobiliario, cambios de combustibles, mineros, y plantas químicas (ver Tabla $\mathrm{N}^{\circ} 10$ ).

\section{TABLA N ${ }^{\circ}$ 10: $\quad$ TIPOS DE PROYECTOS EN QUE SE CONCENTRAN LAS VIOLACIONES}

\begin{tabular}{lccc}
\hline Tipo de proyecto & $\mathrm{CPB}^{\mathrm{a}}$ & $\mathrm{IT}^{\mathrm{b}}$ & Total \\
\hline $\begin{array}{l}\text { Equipamiento: estacionamientos, } \\
\text { estaciones de servicios, terminal de buses, } \\
\text { carreteras, etc. }\end{array}$ & 2 & \\
\hline $\begin{array}{l}\text { Inmobiliario } \\
\text { Cambios de combustibles }\end{array}$ & 1 & 1 & 2 \\
\hline Mineros & 1 & 1 & 1 \\
\hline $\begin{array}{l}\text { Plantas químicas } \\
\text { Saneamiento ambiental (plantas de } \\
\text { tratamiento, rellenos sanitarios, etc.) }\end{array}$ & 10 & & 1 \\
\hline \begin{tabular}{l} 
Total \\
\hline
\end{tabular} & 14 & 2 & 12 \\
\hline
\end{tabular}

a Número de visitas con resultados de cumplimiento parcial bajo

${ }^{\mathrm{b}}$ Número de visitas con resultados de incumplimiento total.

Fuente: Elaboración propia. 
Los proyectos fiscalizados y que mostraron infracciones severas a la RCA se ubican sólo en tres de las treinta y cuatro comunas en que se realizaron visitas de fiscalización; éstas son las comunas de Coronel, Chillán y Los Ángeles.

Adicionalmente decidimos realizar un análisis de reincidencia de infracciones a la RCA. En la Tabla $\mathrm{N}^{\circ} 11$ se presenta una estadística de la reincidencia de transgresiones a la RCA por parte de los proyectos fiscalizados en la región del Bío-Bío bajo el PFAR durante el período 1999-2004. Consideramos aquí que un proyecto reincide en infracción de la RCA si se detecta en violación de las exigencias ambientales cuando es fiscalizado por segunda o más veces.

El número total de proyectos que mostraron reincidencia en incumplimiento fluctúa entre un mínimo de uno durante el año 2001 y un máximo de 14 durante el año 2002 y corresponden al 7\% y 37\% respectivamente de

TABLA N 11 :

ESTADÍSTICA DE REINCIDENCIA DE INFRACCIONES A LAS EXIGENCIAS AMBIENTALES DE LA RCA EN LA REGIÓN DEL BÍO-BÍO: 1999-2004

\begin{tabular}{|c|c|c|c|c|c|c|}
\hline & 1999 & 2001 & 2002 & 2003 & 2004 & Total \\
\hline Proyectos infractores ${ }^{\mathrm{a}}$ & 2 & 14 & 38 & 32 & 17 & 103 \\
\hline Reincidentes $2001^{\mathrm{b}}$ & & 1 & & & & \\
\hline Reincidentes $2002^{\mathrm{c}}$ & & & 14 & & & \\
\hline Reincidentes $2003^{\mathrm{d}}$ & & & & 7 & & \\
\hline Reincidentes $2004^{\mathrm{e}}$ & & & & & 6 & \\
\hline Total infractores reincidentes ${ }^{f}$ & & 1 & 14 & 9 & 10 & 34 \\
\hline
\end{tabular}

${ }^{\text {a }}$ Considera el total de visitas en que se detectaron incumplimientos.

${ }^{b}$ Proyectos que reincidieron en infracciones a la RCA durante el mismo año 2001, es decir, recibieron múltiples visitas durante el mismo año, con resultado de incumplimiento.

c Proyectos que reincidieron en infracciones a la RCA durante el mismo año 2002, es decir, recibieron múltiples visitas durante el mismo año, con resultado de incumplimiento.

d Proyectos que reincidieron en infracciones a la RCA durante el mismo año 2003, es decir, recibieron múltiples visitas durante el mismo año, con resultado de incumplimiento.

e Proyectos que reincidieron en infracciones a la RCA durante el mismo año 2004, es decir, recibieron múltiples visitas durante el mismo año, con resultado de incumplimiento.

${ }^{\mathrm{f}}$ Considera infractores reincidentes del mismo período y aquellos que fueron sorprendidos en incumplimiento durante el período actual y algún período previo en que fueron fiscalizados.

Fuente: Elaboración propia. 
TABLA N ${ }^{\circ}$ 12: $\quad$ ACCIONES PARA INDUCIR CUMPLIMIENTO DE PROYECTOS FISCALIZADOS EN LA REGIÓN DEL BÍO-BÍO, 1999-2004

\begin{tabular}{lcccccc}
\hline Año & $\begin{array}{c}\text { Número de } \\
\text { infractores }^{\mathrm{b}}\end{array}$ & $\begin{array}{r}\text { Número de } \\
\text { infractores } \\
(\text { CB e IT })^{\mathrm{c}}\end{array}$ & $\begin{array}{c}\text { Notificación } \\
\text { escrita }\end{array}$ & $\begin{array}{c}\text { Inicio de } \\
\text { expediente } \\
\text { sancionatorio }\end{array}$ & Multa & $\begin{array}{r}\text { Revocación } \\
\text { de RCA }\end{array}$ \\
\hline 1999 & 2 & 0 & 2 & 0 & 0 & 0 \\
2001 & 14 & 3 & 14 & 3 & 2 & 1 \\
2002 & 38 & 18 & 38 & 1 & 1 & 0 \\
2003 & 32 & 13 & 32 & 1 & 1 & 0 \\
$2004^{\text {a }}$ & 17 & 6 & 17 & 1 & 1 & 0 \\
Total & 103 & 40 & 103 & 6 & 5 & 1 \\
\hline
\end{tabular}

${ }^{a}$ Considera sólo el primer semestre del año.

b Considera visitas de fiscalización con resultados de cumplimiento parcial (bajo, medio, y alto) e incumplimiento total.

${ }^{\mathrm{c}}$ Considera visitas de fiscalización con resultados de cumplimiento bajo e incumplimiento total.

Fuente: Elaboración propia.

los proyectos fiscalizados con incumplimiento. La mayor incidencia de infractores reincidentes del año 2002 coincide con, y podría estar determinada por, el esfuerzo de visitas de fiscalización desplegado durante el período. En efecto, durante el año 2002 se realizó un total de 80 visitas de fiscalización, siendo ésta la mayor cantidad de fiscalizaciones en lo que lleva en operación el PFAR de la región del Bío-Bío.

\section{c) Acciones ejecutadas por el regulador en contra de los infractores}

Finalmente, decidimos explorar las actividades para inducir cumplimiento desarrolladas a partir del resultado de las visitas de fiscalización en aquellos casos en que fueron detectadas infracciones a las RCA de parte de los proyectos aprobados bajo el SEIA. La Tabla No 12 muestra el número de proyectos detectados con algún nivel de incumplimiento y las distintas acciones realizadas por el regulador para inducir mayor cumplimiento ambiental a los transgresores detectados bajo el PFAR de la región del Bío-Bío 
en el período 1999-2004. Las acciones para inducir cumplimiento en aquellos proyectos detectados transgrediendo la RCA incluyen notificación escrita, inicio de un expediente sancionatorio y la aplicación de multas.

La cobertura de notificaciones escritas, según muestran la segunda y cuarta columna de la Tabla $\mathrm{N}^{\circ} 12$, alcanza al $100 \%$ de los proyectos detectados en incumplimiento cada año del período considerado. La apertura de expedientes sancionatorios es más reducida y alcanza solamente a un total de seis casos. Considerando el total de visitas de fiscalización cuyo resultado es un bajo nivel de cumplimiento e incumplimiento total de la RCA, el número de expedientes sancionatorios exhibe una baja cobertura. En efecto, mientras el número total de visitas de fiscalización con resultado de cumplimiento bajo o incumplimiento total alcanza a 40 en el período considerado, la apertura de expedientes afectó sólo al 15\% de los infractores. Adicionalmente, sólo en cinco casos se observa aplicación de multas, lo que representa al $12,5 \%$ de los casos detectados en cumplimiento bajo o incumplimiento total ${ }^{21}$. Finalmente, la revocación de la RCA afectó sólo a un proyecto en el período considerado (ver Tabla $\mathrm{N}^{\circ} 12$, últimas tres columnas).

\section{DISCUSIÓN Y CONCLUSIONES}

El Programa de Fiscalización Ambiental Regional (PFAR) de la región del Bío-Bío se inició de manera preliminar el año 1999, se interrumpió durante el año 2000, para luego, a partir del año 2001, mantener una operación continua. El PFAR es un instrumento que ha permitido hacer seguimiento ambiental a los proyectos de inversión que se han evaluado ambientalmente y que han obtenido la aprobación de la Corema bajo el Reglamento del SEIA introducido en la Ley de Bases del Medio Ambiente de 1994.

Desde el punto de vista del diseño del PFAR, la Conama junto a servicios públicos con competencia ambiental se valen de las visitas de fiscalización para verificar el cumplimiento de las exigencias ambientales establecidas en la RCA. En caso de detectar algún grado de incumplimiento, la Conama procede de acuerdo con lo establecido en el RSEIA, lo cual incluye la elaboración de informes de la situación irregular y la presentación del caso a la Corema, la que resuelve sobre la aplicación de medidas que consideran desde una amonestación escrita hasta la imposición de multas

${ }^{21}$ Las multas aplicadas en los cinco casos mencionados fluctuaron entre un mínimo de 150 UTM en el año 2003 y un máximo de 500 UTM en el año 2001. 
(sanciones monetarias) e incluso la revocación de la RCA, previa apertura y ejecución administrativa de un expediente sancionatorio.

El análisis de este trabajo sugiere que la estrategia de fiscalización aplicada en el PFAR no ha sido suficiente para inducir perfecto cumplimiento ambiental en el período estudiado y se observaron resultados de cumplimiento más bien moderados. Considerando sólo las visitas de fiscalización en que se ha detectado cumplimiento bajo o incumplimiento total, la tasa de infracciones alcanza a un $20 \%$ de las visitas efectuadas.

Desde una perspectiva alternativa es posible afirmar que el 50\% de las visitas resultan en cumplimiento perfecto. La tasa de cumplimiento se eleva hasta un nivel de $80 \%$ si se agregan aquellas visitas con resultado de cumplimiento de la RCA alto o medio. No obstante, es importante mencionar que el referido nivel de cumplimiento debe ser considerado con cautela, debido a que las visitas de fiscalización consisten en general en inspecciones visuales y revisión de bitácoras de operación y ejecución de los proyectos, sin considerar, en los casos pertinentes, el cumplimiento de límites de emisiones, efluentes y niveles de ruido, entre otros. Por lo tanto, el nivel de cumplimiento podría ser incluyo menor.

Los proyectos que presentan mayor grado de transgresiones de la RCA son los de saneamiento ambiental. Estos proyectos se caracterizan en general por el nivel de atención pública que generan, y en algunos casos incluso provocan conflictos con aquellos que se consideran afectados por su ejecución, como también porque en la práctica son los que pueden presentar mayores impactos en contaminación de generarse alguna eventualidad. De acuerdo con nuestro análisis, es en este tipo de proyectos en que se ha concentrado parte importante del esfuerzo de visitas de fiscalización, con alrededor de un $40 \%$ del total del visitas realizadas por el PFAR de la región del Bío-Bío en el período considerado.

En base a los resultados observados y al análisis del PFAR, concluimos que es posible modificar aspectos del diseño de fiscalización bajo el SEIA que permitan mejorar los niveles de cumplimiento ambiental. El sistema de determinación de sanciones y la imposición de éstas reduce el poder disuasivo de las mismas. La determinación de la multa no está basada en una metodología establecida, sino que, por el contrario, es una decisión que toma la Corema caso a caso en base a los antecedentes que se le presentan. En consecuencia, no existe necesariamente una relación conocida ex ante entre el tipo y/o magnitud de la infracción y la sanción, lo que implica, desde el punto de vista de un potencial infractor, la presencia de incerti- 
dumbre respecto a los niveles de las multas a ser aplicadas. Adicionalmente, en el diseño actual, un eventual infractor conoce el nivel máximo de la multa que se le puede aplicar, la que bien podría estar muy por debajo del incentivo para transgredir, lo que se traduce en una clara reducción del poder disuasivo de las sanciones.

El amplio rango para las sanciones monetarias, así como su aplicación mediante un proceso administrativo, generan efectivamente incertidumbre respecto del nivel de éstas. No obstante que el tema referido a cuál es el diseño apropiado de sanciones está fuera del alcance de este trabajo, existen algunas alternativas que pueden ser consideradas como criterios que guíen la definición y aplicación de las mismas. Primero, sería deseable relacionar las sanciones con el daño ambiental generado. Segundo, alternativamente, también puede ser deseable ligar éstas con el beneficio económico obtenido por el incumplimiento. En ambos casos se presentan problemas prácticos de implementación; sin embargo, especificar criterios que sean conocidos por la población regulada puede permitir mejorar la percepción de las sanciones y reducir la incertidumbre de parte de los agentes involucrados - los titulares de los proyectos - respecto de las consecuencias de estar en incumplimiento.

Es interesante mencionar que la fiscalización de las exigencias ambientales de la RCA es coordinada por la Conama, lo que le da transparencia al proceso, debido a que éste es de conocimiento de todos los servicios participantes. Sin embargo, existe un nivel de fiscalización de aspectos sectoriales que hace que el esfuerzo para inducir cumplimiento sea eminentemente de carácter multisectorial, lo cual se traduce de algún modo en superposición de acciones fiscalizadoras que podría ser percibido por la población regulada como una presión excesiva y señales poco claras de parte del (de los) regulador(es). Adicionalmente, más allá del proceso del SEIA, la Conama tiene escaso control sobre el nivel de cumplimiento y fiscalización de la normativa ambiental por parte de las entidades sectoriales. Como consecuencia, este diseño institucional de carácter multisectorial para la fiscalización puede también contribuir a mermar el poder disuasivo de la acción del Estado contra los infractores, imponiendo barreras para el logro de niveles adecuados de cumplimiento ${ }^{22}$.

${ }^{22}$ El diseño institucional de fiscalización multisectorial en Chile fue cuestionado recientemente en el informe OCDE-CEPAL, 2005, en el que se concluye que "una política de fiscalización de la normativa ambiental sobre la base de la coordinación de los órganos sectoriales de supervisión y control no es la solución institucional más eficaz para asegurar su cumplimiento”, OCDE-CEPAL, 2005, p. 128. 
El análisis del perfil de los proyectos infractores a la RCA sugiere que éstos se concentran en determinadas áreas geográficas y corresponden a aquellos de menor antigüedad, principalmente proyectos de saneamiento ambiental. Es precisamente en proyectos que tienen esta última característica que se ha focalizado la actividad fiscalizadora, pues concentran el 39,7\% de la muestra analizada.

El PFAR es restringido debido a los escasos recursos económicos que se le destinan. La restricción de recursos y la diversidad de ámbitos de acción de la Conama en la región del Bío-Bío constituyen un impedimento para su labor fiscalizadora, reduciendo de paso su poder disuasivo. Considere, a manera de ejemplo, que la Dirección Regional de la Conama en la región del Bío-Bío cuenta con sólo una camioneta para un total de treinta profesionales. Un mayor nivel de recursos permitiría ampliar el PFAR a fin de conocer el comportamiento ambiental de parte de los proyectos que no han sido fiscalizados. Recursos adicionales al PFAR podrían ampliar el ámbito de las visitas de fiscalización, más allá de visitas inspectivas de tipo visual, permitiendo iniciar de manera sistemática evaluaciones cuantitativas respecto del cumplimiento de normas relativas, por ejemplo, a emisiones, efluentes y niveles de ruido. Ello sería posible si se dispusiera de los equipos necesarios para toma de muestras y mediciones in situ de las variables ambientales.

Propender a una mayor sistematización tanto de la información que se obtiene en las visitas inspectivas como también de su procesamiento y análisis puede fortalecer la labor fiscalizadora. En este sentido no es clara, desde el punto de vista de procedimientos, la situación de cumplimiento que logra cada proyecto. Al respecto, sería deseable el diseño de fichas de fiscalización que den cuenta de los principales aspectos del proyecto que se observa en cada visita de inspección, a fin de que en la misma visita de fiscalización se constate el estatus de cumplimiento ambiental del proyecto. Nuestro trabajo ofrece precisamente una propuesta específica al respecto, al definir y aplicar estatus de cumplimiento sobre proyectos previamente fiscalizados.

Finalmente, nuestro trabajo sugiere que es posible mejorar el diseño de fiscalización para inducir cumplimiento ambiental. El análisis de la fiscalización y los resultados de cumplimiento pueden contribuir a mitigar los impactos ambientales de proyectos productivos que potencien el desarrollo económico del país. 


\section{ANEXO 1}

TABLA A-1:

CUMPLIMIENTO AMBIENTAL EN COMUNA DE LOS ÁNGELES SEGÚN TIPO DE PROYECTO: 1999-2004

\begin{tabular}{|c|c|c|c|c|c|c|}
\hline Tipos de proyectos & $\mathrm{CT}^{\mathrm{a}}$ & $\mathrm{CPA}^{\mathrm{b}}$ & $\mathrm{CPM}^{\mathrm{c}}$ & $\mathrm{CPB}^{\mathrm{d}}$ & $\mathrm{IT}^{\mathrm{e}}$ & Total \\
\hline Inmobiliarios & 2 & & & & & 2 \\
\hline Mineros & 1 & 2 & 1 & 1 & & 5 \\
\hline Plantas químicas & 2 & 1 & & & & 3 \\
\hline Forestales & 3 & & 1 & & & 4 \\
\hline Acuícolas & & 1 & 2 & & & 3 \\
\hline $\begin{array}{l}\text { Saneamiento ambiental (plantas de } \\
\text { tratamiento, rellenos sanitarios, etc.) }\end{array}$ & 2 & & & 4 & & 6 \\
\hline Total & 10 & 4 & 4 & 5 & 0 & 23 \\
\hline
\end{tabular}

a Número de visitas con resultados de cumplimiento total.

b Número de visitas con resultados de cumplimiento parcial alto.

c Número de visitas con resultados de cumplimiento parcial medio.

${ }^{\mathrm{d}}$ Número de visitas con resultados de cumplimiento parcial bajo.

${ }^{\text {e }}$ Número de visitas con resultados de incumplimiento total.

Fuente: Elaboración propia.

TABLA A-2:

CUMPLIMIENTO AMBIENTAL EN COMUNA DE CHILLÁN SEGÚN TIPO DE PROYECTO: 1999-2004

\begin{tabular}{lcccccc}
\hline Tipos de proyectos & $\mathrm{CT}^{\mathrm{a}}$ & $\mathrm{CPA}^{\mathrm{b}}$ & $\mathrm{CPM}^{\mathrm{c}}$ & $\mathrm{CPB}^{\mathrm{d}}$ & $\mathrm{IT}^{\mathrm{e}}$ & Total \\
\hline $\begin{array}{l}\text { E (Equipamiento: estacionamientos, } \\
\text { estaciones de servicios, terminal de } \\
\text { buses, carreteras, etc.) }\end{array}$ & 1 & & & & & \\
\hline $\mathrm{G}$ (Inmobiliarios) & 2 & 2 & & & & 1 \\
\hline $\mathrm{H}$ (Cambios de combustibles) & 2 & 2 & & & 1 & 2 \\
\hline I (Mineros) & 1 & & & & 1 \\
\hline K (Plantas químicas) & 11 & 8 & 2 & 3 & 2 & 26 \\
\hline $\begin{array}{l}\text { O (Saneamiento ambiental: plantas de } \\
\text { tratamiento, rellenos sanitarios, etc.) }\end{array}$ & 6 & 2 & 2 & 3 & & 13 \\
\hline \begin{tabular}{l} 
Total \\
\hline
\end{tabular} & 11 & & & & & \\
\hline
\end{tabular}

a Número de visitas con resultados de cumplimiento total.

b Número de visitas con resultados de cumplimiento parcial alto.

${ }^{\mathrm{c}}$ Número de visitas con resultados de cumplimiento parcial medio.

${ }^{\mathrm{d}}$ Número de visitas con resultados de cumplimiento parcial bajo.

e Número de visitas con resultados de incumplimiento total.

Fuente: Elaboración propia. 


\section{REFERENCIAS BIBLIOGRÁFICAS}

Cohen, Mark: "Monitoring and Enforcement of Environmental Policy". En Henk Folmer y Tom Tietenberg (eds.), The International Yearbook of Environmental and Resource Economics 1999/2000, A Survey of Current Issues. Edward Elgar, 1999.

Comisión Nacional del Medio Ambiente, Región del Bío-Bío. Informe de Gestión Ambiental, 2002.

Dasgupta, Susmita, Hemamala Hettige y David Wheeler: "What Improves Environmental Compliance? Evidence from Mexican Industry”. En Journal of Environmental Economics and Management, № 39 (2000), pp. 39-66.

Diario Oficial de la República de Chile. "Decreto Supremo No 30”, marzo de 1997, modificado por el Decreto Supremo No 95, agosto de 2001.

Heyes, Anthony: "Implementing Environmental Regulation: Enforcement and Compliance”. En Journal of Regulatory Economics, Vol. 17 No 2 (2000), pp. 107129.

Organización de Cooperación y Desarrollo Económico (OCDE) y Comisión Económica para América Latina y el Caribe (CEPAL): Evaluaciones del Desempeño Ambiental, Chile, 2005.

Palacios, Milagros, y Carlos Chávez: "Programa de Compensación de Emisiones: Evaluación del Diseño de Fiscalización y su Cumplimiento”. En Estudios Públicos No 88 (primavera 2002), pp. 97-126.

"Determinants of Compliance in the Emissions Compensation Program in Santiago, Chile”. En Environment and Development Economics, № 10 (2005), pp. 453-483.

Ruiz-Tagle, María Teresa: "What are the Determinants of Environmental Compliance in the Chilean Manufacturing Industry? A Case Study". Environmental Economy and Policy Research Discussion Paper Series Number 17, Department of Land Economy, University of Cambridge, 2006.

U. S. Environmental Protection Agency (EPA) (Agencia de Protección Ambiental de los Estados Unidos): Principles of Environmental Enforcement. Office of Enforcement, 1992.

World Bank Policy Research Report: "Regulando la Contaminación en el Mundo Real”, capítulo 2. En Armonización de la Actividad Industrial con el Medio Ambiente: Nuevas Funciones de la Comunidad, el Mercado y el Gobierno. Alfaomega S. A., 2000. 